\title{
Cellular cohesion as a prognostic factor in malignant melanoma: a retrospective study with up to 12 years follow-up
}

\author{
Ioannis Roxanis and Jade Chow
}

Department of Cellular and Molecular Medicine, St George’s Hospital Medical School, Tooting, London, UK

\begin{abstract}
Malignant melanomas have a high metastatic potential. Although the depth of tumour invasion is the single most important histological prognostic factor, in clinical practice this correlation is frequently challenged. In this study, we assessed the cohesion of malignant melanocytes in the dermal component of all primary melanomas in vertical growth phase with tumour thickness $>0.76 \mathrm{~mm}$ diagnosed in our Department between 1990 and 1995. The rationale behind this morphological evaluation was based on the hypothesis that a change in the adhesion molecule profile of melanoma cells may manifest visually discrete changes in the way that cells group together in dermal aggregates. We used a dyscohesion score based on the proportion of invasive tumour occupied by dyscohesive neoplastic cells and assessed its clinical significance by correlating it with the incidence of recurrence, regional and distant metastases and survival of the patients. Follow-up was up to 12 years. We found that the degree of melanoma cell dyscohesion was associated with the probability of local recurrence or metastasis. This correlation was particularly significant when dyscohesion involving an area smaller than $25 \%$ (dyscohesion score 1 ) of the dermal component was compared to dyscohesion involving a larger area (scores 2-4). Melanomas in the latter group had significantly increased likelihood of recurrence or metastasis $(P<0.025$, log-rank test). This was particularly the case in T1-T3 melanomas $(P<0.005)$. Similarly, T1-T3 melanomas with dyscohesion score 1 had a significantly higher survival rate $(P<0.025)$. In the same cohort, both disease-free survival and survival were not significantly correlated to thickness, probably due to the limited number of cases. Finally, we showed that extent of dyscohesion was independent of Breslow thickness or tumour regression. We believe that estimation of melanoma cell dyscohesion is a reliable histological prognostic factor that may be appropriate in clinical practice.
\end{abstract}

Modern Pathology (2010) 23, 502-510; doi:10.1038/modpathol.2009.171; published online 19 February 2010

Keywords: melanoma; cellular cohesion; metastasis; prognosis

Malignant melanoma is by far the cutaneous tumour with the highest metastatic potential. ${ }^{1}$ Despite increased public awareness promoting early detection of malignant melanoma, ${ }^{2}$ its mortality rate remains high, surpassed only by lung cancer (reviewed by Slominski et $a l^{3}$ ).

It is now well established that, in the majority of cases, a malignant melanoma acquires its metastatic potential through the successive steps of dysplasia, melanoma in situ, radial (horizontal) growth phase and vertical growth phase. In fact, radial growth

Correspondence: Dr I Roxanis, Department of Cellular Pathology, King's College Hospital NHS Foundation Trust, Denmark Hill, London SE5 9RS, UK.

E-mail: ioannis.roxanis@kch.nhs.uk

Received 19 May 2009; revised 30 June 2009; accepted 1 July 2009; published online 19 February 2010 phase melanomas are considered practically not to have metastatic potential, regardless of level of invasion or thickness, ${ }^{4,5}$ except for extremely rare cases of invasive radial growth phase melanomas where occasional tumour cells extend into the upper dermis, singly or in small nests. ${ }^{6}$ However, the same is practically true for vertical growth phase melanomas less than $0.76 \mathrm{~mm}$ in thickness that metastasize only very rarely. ${ }^{7}$ Indeed, the thickness of invasion into dermis (Breslow thickness) is the single most important histological prognostic factor. This, together with ulceration and the level of dermal invasion (Clark level), is the only histopathological feature incorporated into the new TNM classification of the American Joint Committee on Cancer. ${ }^{8}$ Other reported histopathological prognostic parameters include regression of the dermal tumour mass, vascular invasion, neurotropism and number of 
tumour-infiltrating lymphocytes. ${ }^{5}$ Histopathological features with prognostic significance represent static reflections of functional characteristics. In this context, Breslow thickness represents the capacity of melanoma cells to invade through the surrounding extracellular matrix components, and ulceration represents their ability to breach the overlying epidermis.

It is becoming increasingly clear that tumour progression to metastasis is a complex process, involving numerous homotypic and heterotypic interactions between the neoplastic cells and their environment. In order to acquire metastatic potential, the neoplastic cells have to detach from their original neighbours and migrate through the surrounding extracellular matrix before entering lymphatics and blood vessels. ${ }^{9}$ The progression of malignant melanoma from in situ to metastatic disease has been studied both in vitro and in vivo, and some molecular interactions underlying this process have been clarified. Melanoma cells have been shown to exhibit altered expression of intercellular adhesion molecules that reflect their disturbed cell-cell interactions with an initial loosening of their adhesion to their neighbours. ${ }^{10}$ Cytological smear preparations obtained from patients with metastatic melanoma are composed of loosely cohesive or noncohesive cells with only occasional cohesive clusters seen. ${ }^{11}$

Although a variety of patterns of dermal invasion of melanoma cells have been described ${ }^{12,13}$ and demonstrated to show prognostic significance, ${ }^{14}$ there are no reports on morphological assessment of tumour cohesion in situ. In this study, we assessed, semi-quantitatively, the extent of cell-cell cohesion on paraffin sections of invasive melanomas. The rationale behind this morphological evaluation was based on the hypothesis that the altered adhesion molecule profile of melanoma cells may manifest changes in the way that cells group together in dermal aggregates. We used a dyscohesion score based on the proportion of the tumour mass occupied by dyscohesive neoplastic cells. The functional and clinical significance of tumour cell dyscohesion was then assessed by correlating the degree of tumour cell cohesion with the incidence of recurrence, regional and distant metastases and survival of the patients.

\section{Materials and methods}

\section{Patients}

We reviewed all cases diagnosed with a primary cutaneous malignant melanoma at the Histopathology Department of St George's Hospital between 1990 and 1995. In total, 188 cases were identified. From these, 34 cases were in situ melanomas, 52 were in radial growth phase and 102 cases were in vertical growth phase. According to established criteria, vertical growth phase is characterized by dermal clusters of melanoma cells larger than junctional nests or larger than 20-25 cells across, with mitoses usually present. ${ }^{3}$ Because the aim of this study was to evaluate morphologically the cohesion between malignant cells in melanomas with metastatic potential, we focused our attention to the latter category. The reason for this is that melanomas in radial growth phase are practically considered to possess no metastatic potential. ${ }^{4}$ In addition, because most patients with very thin melanomas in vertical growth phase (Breslow thickness $\leq 0.75$ ) are cured by surgical excision, ${ }^{7,15}$ we also excluded seven such cases from this study. Therefore, only 95 cases were selected for further histological evaluation and follow-up.

During histological evaluation seven further cases were excluded for several reasons: one case was diagnosed as desmoplastic melanoma, one case as naevoid/minimal deviation melanoma and one case as spitzoid melanoma. For biopsy and laboratory technical reasons, tumours could not be fully assessed in three other cases: in one case a central prominent part of the melanoma was missing, in one case the maximum thickness could not be assessed and in one case there were extensive areas of necrosis and haemorrhage. Finally, in one case it was not possible to confirm that the tumour was a primary.

\section{Follow-Up}

Follow-up data were obtained from patients' files retrieved from the medical records of St George's Hospital. Thirteen cases were excluded during this phase: in nine cases medical notes were missing despite multiple efforts to retrieve them and in one case no follow-up data were included in the notes. Finally, three cases were excluded because the patients moved away very shortly after the excision of their melanoma. Therefore, the final yield consisted of 75 cases of vertical growth phase primary cutaneous malignant melanomas.

The main source of follow-up data was the notes of the Melanoma Clinic of St George's Hospital where patients with excised melanomas are followed up. To supplement our follow-up further to the information provided by the Melanoma Clinic, we sent letters to their general practitioners for 25 cases. We obtained replies in 15 cases, considerably extending our follow-up data. As a result, 51 of the studied patients had a complete follow-up. Of these, 16 were followed up to the time when this study was completed (1 patient for 12 years, 5 for 11, 4 for 10,1 for 9,1 for 8 and 4 for 7; mean 9.5 years, median 10 years) and 35 were followed up to the time of their death (mean 2.86 years, median 2 years). The remaining 24 patients had an incomplete follow-up. From the latter group, 3 patients had a $0-2$ years follow-up, 6 had a 3-4 years follow-up and 15 had a 5-9 years follow-up (mean 5.17 years, median 
5 years). Overall, the range of follow-up period was 0-12 years with a mean of 5.01 years and a median of 5 years.

\section{Histological Assessment}

We assessed the following histological parameters in each case:
a. Breslow thickness
b. Presence or absence of ulceration
c. Presence or absence of regression

\section{Assessment of Cohesion}

Cellular cohesion was assessed in conventional paraffin sections stained with haematoxylin and eosin. The cohesion of adjacent melanoma cells was considered only in the invasive dermal part of the tumours because it is exclusively cells of this component that have been shown to possess metastatic potential. ${ }^{4}$

Adjacent melanoma cells were considered to be dyscohesive when the distance between them was greater than the diameter of the cells (Figure 2b). When the distance was less, we regarded it as artefactual cytoplasmic retraction due to specimen processing (Figure 1b, arrows). A helpful feature that was used for the distinction between dyscohesion and artefactual cytoplasmic retraction was the way of arrangement of the cell membranes of adjacent cells. Cell membranes of neighbouring dyscohesive cells were randomly arranged. In addition, dyscohesive melanoma cells frequently showed cytoplasmic processes (Figure 2b). In contrast, neighbouring cells with a gap between them but with a parallel arrangement of their cell membranes were generally considered to have undergone artefactual cytoplasmic retraction (Figure $1 \mathrm{~b}$, arrows). Areas of melanomas composed of singly dispersed cells within dermis were also considered as dyscohesive.

To quantify the degree of dyscohesion, we devised a dyscohesion score based on the proportion of the dermal component occupied by dyscohesive melanoma cells. Hence, melanomas in which $0-25 \%$ of the dermal component was composed of dyscohesive cells received a dyscohesion score 1. Dyscohesion score 2 corresponded to dyscohesion involving $25-50 \%$ of the dermal component (Figure 1a), score 3 was given to tumours with 50-75\% dermal proportion of dyscohesion and melanomas of which $75-100 \%$ of the dermal component was dyscohesive were given score 4 (Figure 2a). All the histological sections were assessed by one person (IR). In addition, 20 randomly assessed cases were re-evaluated by another observer (JC), without knowledge of the original score.

\section{Statistical Analysis}

Kaplan-Meier survival curves were compared by the log-rank test. An estimate of the differences was obtained with the hazard ratio (the risk of matastasis/death in one group to the risk of metastasis/ death to the other group).

Contingency tables were analysed using the $\chi^{2}$-test with the appropriate use of Fisher's exact test in smaller samples.

Kendall's rank correlation coefficient was used for the study of relation of dyscohesion and tumour thickness.
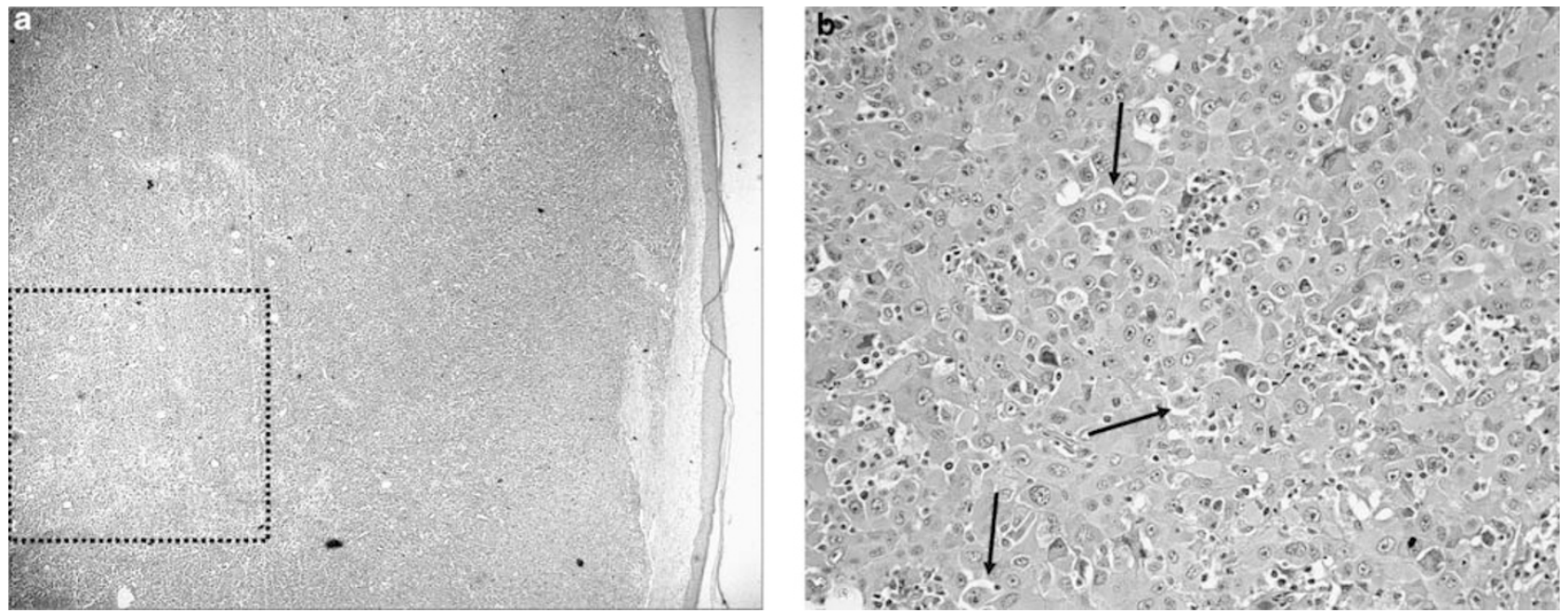

Figure 1 Haematoxylin and eosin $(\mathrm{H}+\mathrm{E})$ stained section from a $\mathrm{T} 4$ (Breslow thickness $35 \mathrm{~mm}$ ) melanoma present for $21 / 2$ years. The lesion was excised from the left upper arm of a 74-year-old male. During 5 years of follow-up, there was no evidence of local recurrence or distant metastasis. The tumour was given a dyscohesion score 2. Areas of dyscohesion are seen in the dotted part of the section (a). Cohesive areas are shown in higher magnification in $\mathbf{b}$. Arrows in $\mathbf{b}$ indicate artefactual cytoplasmic retraction rather than genuine dyscohesion. 

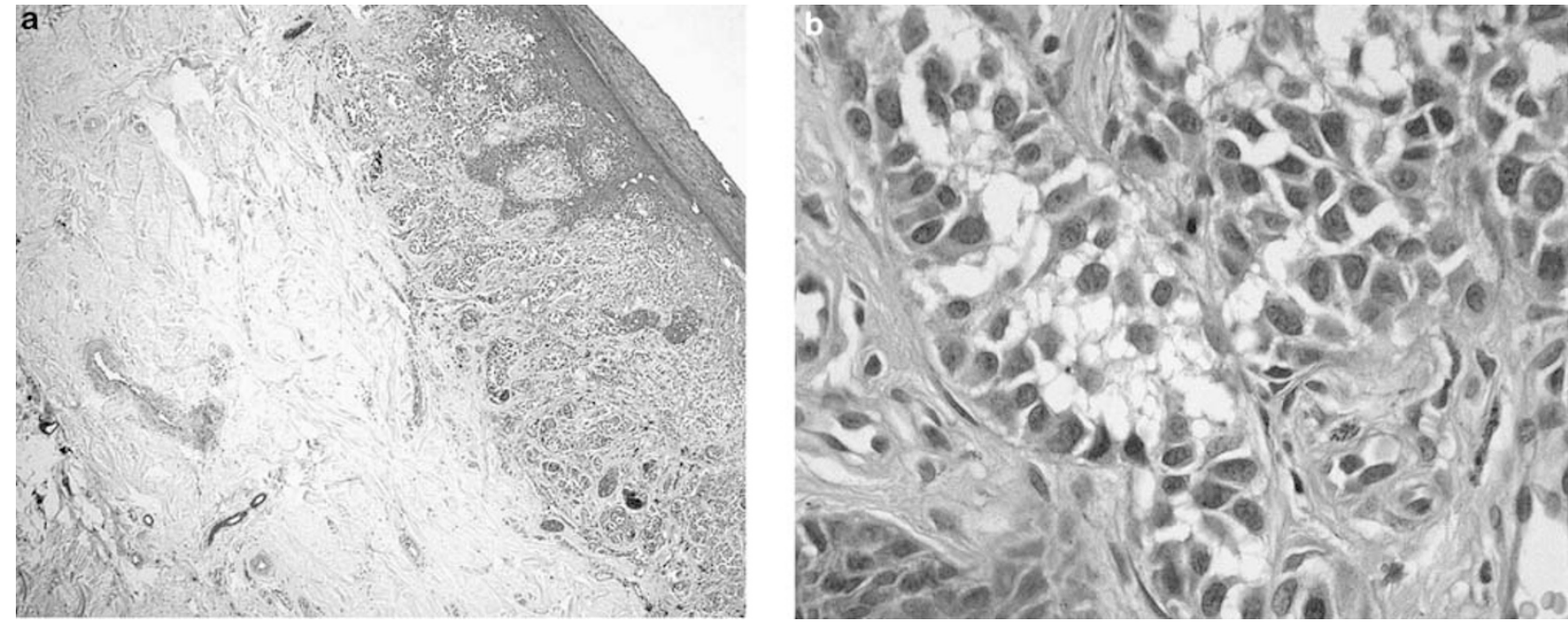

Figure 2 Haematoxylin and eosin $(\mathrm{H}+\mathrm{E})$ stained section from a T1 (Breslow thickness $1 \mathrm{~mm}$ ) melanoma excised from the left foot of a 49year-old female. The tumour was given a dyscohesion score 4. Despite its low thickness, melanoma deposits on the left thigh were detected 2 years after the excision, lung metastasis in 4 years and the patient died within 5 years of presentation. Panel a Shows that the pattern of dermal infiltration is nested. In $\mathbf{b}$, higher magnification shows genuine dyscohesion between the melanoma cells with gaps exceeding the cells' diameter and presence of cytoplasmic processes.

Inter-observer variation in the assessment of dyscohesion was analysed by $\kappa$ statistics.

\section{Results}

\section{Clinical Information}

There were 24 men and 51 women, with an age range of 19 and 93 years at the time of excision (mean 59.4 years). The Breslow thickness ranged from 0.8 to $35 \mathrm{~mm}$ (mean $3.9 \mathrm{~mm}$ ). The anatomic location was recorded in 71 cases. Fifty of them were located in the extremities, 10 were located in the head, 10 were located in the trunk and 1 case was a vulval melanoma.

\section{Dyscohesion Score}

All cases were classified according to the final version of the American Joint Committee on Cancer Staging System for Cutaneous Melanomas ${ }^{5}$ (T1 $\leq 1 \mathrm{~mm}$, T2 between 1.01 and $2 \mathrm{~mm}$, T3 between 2.01 and $4 \mathrm{~mm}$ and $\mathrm{T} 4>4 \mathrm{~mm}$ ). Of them, 11 were T1 tumours, 19 were T2, 22 were T3 and 23 were $\mathrm{T} 4$.

As shown in Table 1, the majority of the studied cases had a dyscohesion score 1 (48\%), with a relatively even distribution among T1 (45\%), T2 (59\%), T3 $(42 \%)$ and T4 (48\%) tumours. Thinner tumours (T1 and T2) had a tendency to show higher dyscohesion score (27 and $24 \%$, respectively, had dyscohesion score 4) than thicker (T3 and T4) ones (13\% each had dyscohesion score 4 ), although this difference was not statistically significant.
Table 1 Dyscohesion score and tumour thickness

\begin{tabular}{lccccc}
\hline Dyscohesion & \multirow{2}{*}{ T1 } & T2 & T3 & T4 & Total \\
\hline 1 & $5(45 \%)$ & $10(59 \%)$ & $10(42 \%)$ & $11(48 \%)$ & $36(48 \%)$ \\
2 & $2(18 \%)$ & $2(12 \%)$ & $3(13 \%)$ & $5(22 \%)$ & $12(16 \%)$ \\
3 & $1(9 \%)$ & $1^{*}(6 \%)$ & $8^{*}(33 \%)$ & $4(17 \%)$ & $14(19 \%)$ \\
4 & $3(27 \%)$ & $4(24 \%)$ & $3(13 \%)$ & $3(13 \%)$ & $13(17 \%)$ \\
Total & 11 & 17 & 24 & 23 & 75
\end{tabular}

*The only significant difference seen was in dyscohesion 3 subgroup (more T3 than T2 cases; $P=0.04$, Fisher's exact test).

\section{Melanoma Thickness, Ulceration and Regression}

Breslow thickness has been shown to be the single most important histological prognostic parameter. The disease-free Kaplan-Meier survival curves in T1-T4 melanomas are shown in Figure 3a. Absence of in-transit metastases, satellite metastases, local recurrence (after complete excision of the primary) and nodal or visceral metastases were all considered in disease-free survival. Disease-free times were calculated from the time of excision of the primary melanoma. As expected, the disease-free survival rates showed a gradual decline from $\mathrm{T} 1$ to $\mathrm{T} 4$ melanomas (Figure 3a). However, probably because of the relatively small number of cases in our study, this difference did not reach statistical significance (log-rank test).

Similarly, the survival rates of T1-T4 melanomas showed no statistically significant decline (Figure $3 b)$. It should be noted that survival rate was estimated taking melanoma-related deaths only into consideration. 

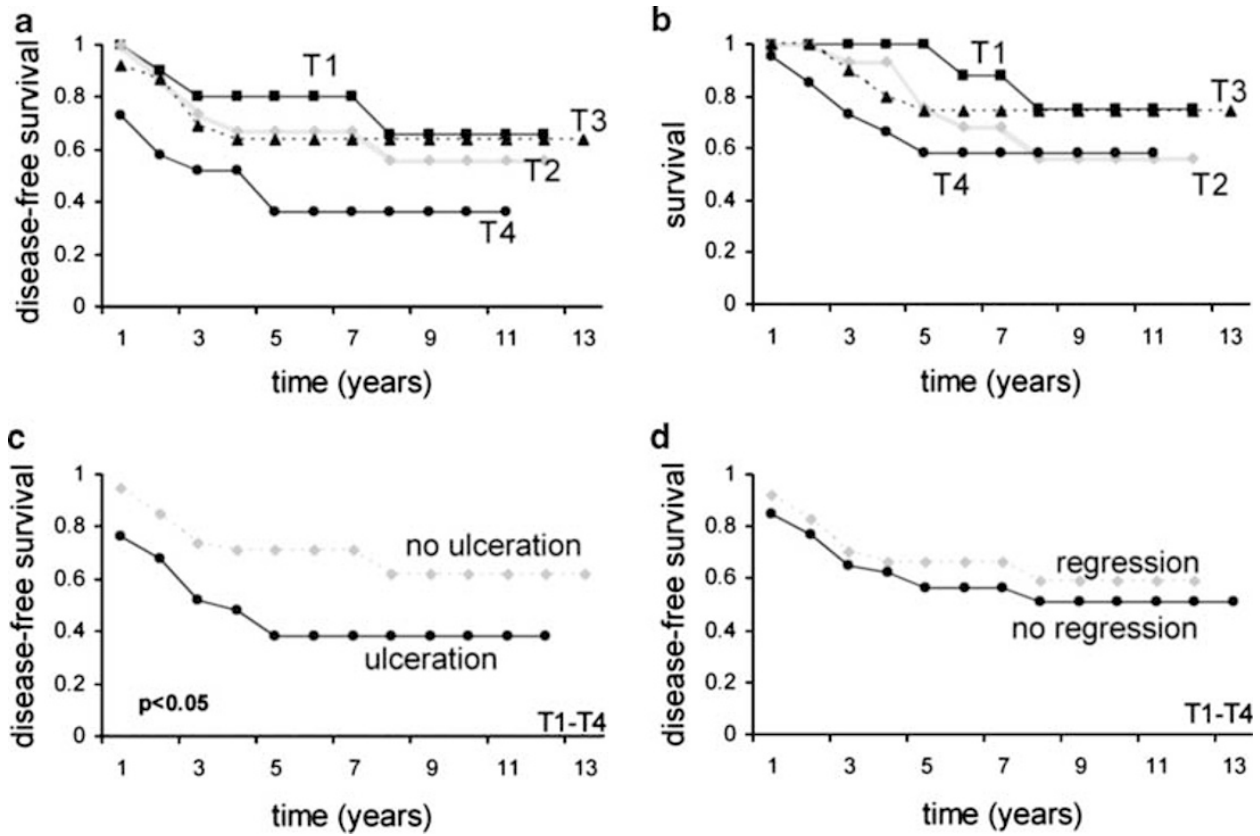

Figure 3 (a) Kaplan-Meier disease-free survival curves of T1-T4 melanomas. The differences were not significant (log-rank test). (b) Kaplan-Meier survival curves of T1-T4 melanomas. The differences were not significant (log-rank test). (c) Kaplan-Meier disease-free survival curves of 72 melanomas comparing lesions with or without ulceration. The difference was significant $(P<0.05$, log-rank test). (d) Kaplan-Meier disease-free survival curves of all 75 melanomas comparing lesions with or without regression. The difference was not significant (log-rank test).

Ulceration was present in 30 out of 72 cases that could be evaluated. Ulcerated melanomas had a significantly lower disease-free survival rate compared to non-ulcerated tumours $(P<0.05$, log-rank test, $\chi^{2}=4.29$ ) (Figure 3c).

Regression was noted in 27 out of the 75 cases. In our sample, the disease-free survival curves of patients with melanomas with or without regression were close, showing no statistically significant difference (Figure 3d).

\section{Dyscohesion and Disease-Free Survival}

We then progressed to the analysis of the prognostic significance of cellular dyscohesion. We first plotted the metastasis-free Kaplan-Meier curves of all 75 melanomas in the four groups of dyscohesion (scores 1-4). As shown in Figure 4a, the diseasefree survival rates of melanomas with dyscohesion scores 2, 3 and 4 were almost indistinguishable, whereas the corresponding rate of melanomas with dyscohesion score 1 was clearly higher.

Therefore, we pooled the groups with dyscohesion scores 2, 3 and 4 together, creating two subgroups: The first had dyscohesion in less than $25 \%$ of the dermal component of the tumour (subgroup with dyscohesion score 1-low dyscohesion subgroup) and the second had dyscohesion in more than $25 \%$ of the dermal component of the tumour (subgroup with dyscohesion scores 2, 3 and 4-high dyscohesion subgroup). As shown in Figure $4 \mathrm{~b}$, the disease-free rates of these two subgroups showed a significant difference $\left(P<0.025\right.$, log-rank test, $\left.\chi^{2}=6.1\right)$. The hazard ratio (the risk of recurrence or metastasis in the low dyscohesion subgroup to the risk of recurrence or metastasis in the high dyscohesion subgroup) was 0.38 .

Interestingly, the difference in the disease-free rate between these two subgroups in independently studied T1-T4 melanomas (Figure 5) was significant only in T1 and T2 melanomas (Figures 5a and b). In addition, the hazard ratios were increasingly higher going from $\mathrm{T} 1$ to $\mathrm{T} 4$ melanomas (T1, 0; T2, 0.21; $\mathrm{T} 3,0.2$ and $\mathrm{T} 4,0.9)$. When $\mathrm{T} 4$ melanomas were excluded from analysis, the difference of diseasefree survival rates between the low and the high dyscohesion score subgroups showed an even higher level of statistical significance $(P<0.005$, log-rank test, $\chi^{2}=8.207$ ) with a hazard ratio of 0.2 (Figure $4 \mathrm{c}$ ).

\section{Dyscohesion and Melanoma-Related Survival}

The Kaplan-Meier survival curves for all 75 melanomas studied in the four groups of dyscohesion are shown in Figure 6a. Patients with dyscohesion scores 1 and 2 showed similar survival rates, which were higher than survival rates of patients with dyscohesion scores 3 and 4. Comparison of the survival curves of subgroups with dyscohesion score 1 versus dyscohesion scores 2-4 showed no statistical difference (Figure 6b). However, when only T1-T3 melanomas were analysed (Figure 6c) 


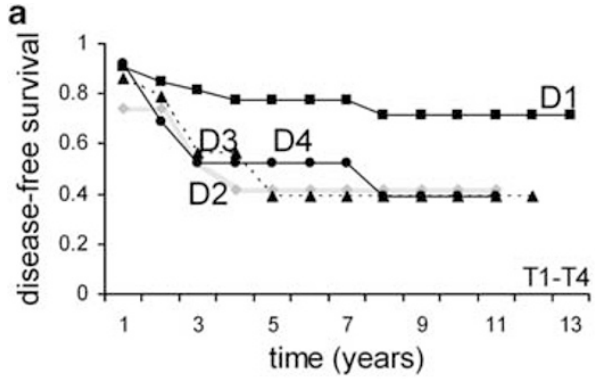

b
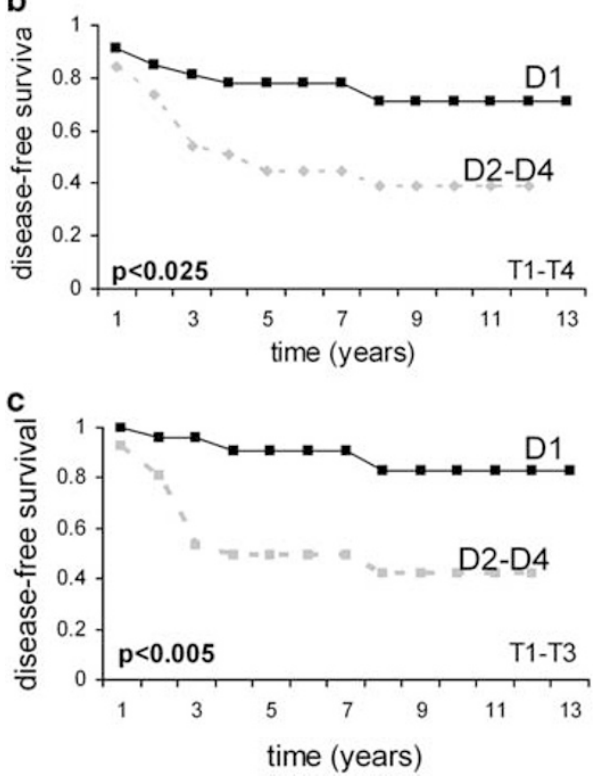

Figure 4 (a) Kaplan-Meier disease-free survival curves of all 75 melanomas comparing lesions with dyscohesion scores 1-4 (D1-D4). (b) Kaplan-Meier disease-free survival curves of all 75 melanomas comparing lesions with dyscohesion score 1 (D1) with lesions with dyscohesion scores 2-4 (D2-D4) pooled together. The difference was significant $(P<0.025$, log-rank test). (c) Kaplan-Meier disease-free survival curves of T1-T3 melanomas only comparing lesions with dyscohesion score 1 (D1) with lesions with dyscohesion scores 2-4 (D2-D4) pooled together. The difference was significant $(P<0.005$, log-rank test).

the difference was significant at the level of $P<0.025$ (log-rank test, $\chi^{2}=5.1$ ).

\section{Relationship of Dyscohesion with Other Prognostic Parameters}

Table 1 shows the distribution of dyscohesion groups in T1-T4 melanomas. As is apparent from the proportion of each dyscohesion score group in the different $\mathrm{T}$ groups, there is no evidence that dyscohesion and tumour thickness are related. This was confirmed statistically using Kendall's rank correlation coefficient.

Dyscohesion was also shown to be unrelated to regression. Indeed, the distribution of different dyscohesion score groups in cases showing regression was almost identical to cases showing no regression (score 1, 48 versus 48\%; score 2, 19 versus $15 \%$; score 3,22 versus $17 \%$ and score 4,11 versus $21 \%$ in melanomas with or without regression correspondingly).

Finally, there was no association between ulceration and dyscohesion score apart from those with score $3(P<0.05)$ (score 1,40 versus $55 \%$; score 2,17 versus $14 \%$; score 3 , 30 versus $10 \%$ and score 4,13 versus $21 \%$ in melanomas with or without ulceration correspondingly).

\section{Dyscohesion Score: Inter-observer Variation}

From the randomly selected cases that were evaluated by a second observer, only 3 out of 20 received a score that would result in different dyscohesion subgroup (low instead of high dyscohesion subgroup or vice versa). The $\kappa$ value was 0.69 (substantial strength of agreement). In 14 out of 20 cases the score was perfectly matched, in 2 out of 20 the score differed by 1 (4 versus 3 and 3 versus 2) and in 1 out of 20 it differed by 2 ( 4 versus 2 ).

\section{Discussion}

In this study, we assessed morphologically the cohesion of malignant melanocytes in the dermal component of potentially metastatic cutaneous melanomas. We noticed that the proportion of dyscohesive cells was variable and, to obtain a quantitative estimate of this proportion, a 'dyscohesion score' was devised. On the basis of the fact that a well-described step in tumour progression is loss of cell-cell contact, and hypothesizing that such loss might be discerned morphologically in paraffin sections as dyscohesion between tumour cells, we evaluated the prognostic significance of dyscohesion score.

Our main finding was that the extent of melanoma cell dyscohesion was associated with the probability of local recurrence or metastasis. This correlation was particularly significant when dyscohesion involving an area smaller than 25\% (defined as dyscohesion score 1) of the dermal component was compared to dyscohesion involving a larger area (dyscohesion scores 2-4). Melanomas with high dyscohesion scores had significantly increased likelihood of recurrence or metastasis. This was particularly the case in T1-T3 melanomas. Similarly, T1-T3 melanomas with dyscohesion score 1 had a significantly higher survival rate. In the same sample, although both disease-free survival and survival were clearly related to thickness, the differences were not statistically significant. Finally, we showed that extent of dyscohesion was not related to Breslow thickness or tumour regression.

There is good evidence that dissociation of malignant cells from their neighbours is the very initial step of tumour progression. ${ }^{16}$ Indeed, during 

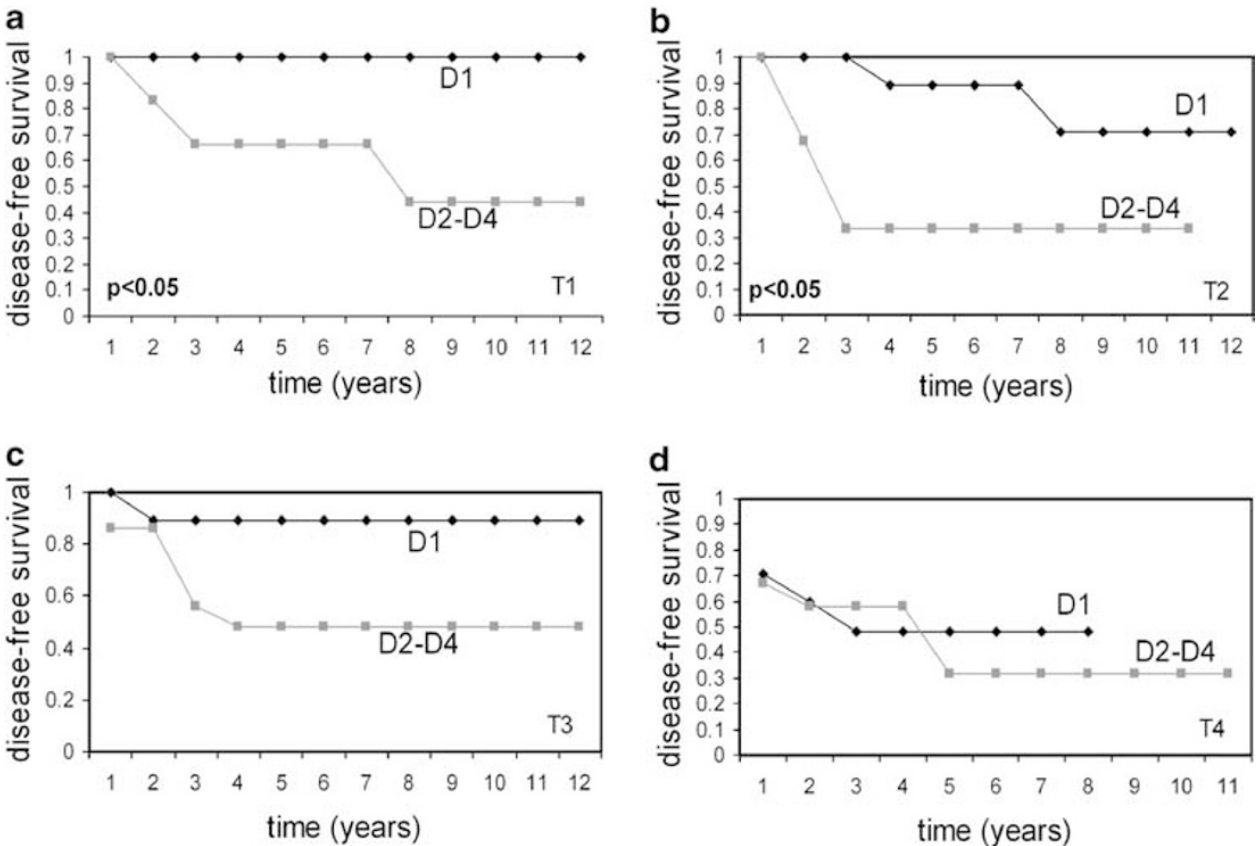

Figure 5 (a) Kaplan-Meier disease-free survival curves of T1 melanomas comparing lesions with dyscohesion score 1 (D1) with lesions with dyscohesion scores 2-4 (D2-D4) pooled together. The difference was significant $(P<0.05$, log-rank test). (b) Kaplan-Meier disease-free survival curves of T2 melanomas comparing lesions with dyscohesion score 1 (D1) with lesions with dyscohesion scores 2-4 (D2-D4) pooled together. The difference was significant $(P<0.05$, log-rank test). (c) Kaplan-Meier disease-free survival curves of T3 melanomas comparing lesions with dyscohesion score 1 (D1) with lesions with dyscohesion scores 2-4 (D2-D4) pooled together. The difference was not significant. (d) Kaplan-Meier disease-free survival curves of T4 melanomas comparing lesions with dyscohesion score 1 (D1) with lesions with dyscohesion scores 2-4 (D2-D4) pooled together. The difference was not significant.

melanoma development, malignant melanocytes initially loosen their adhesion to basal keratinocytes. ${ }^{10}$ Furthermore, homotypic cell-cell adhesion at the site of primary tumour suppresses invasion. ${ }^{9}$ In this context, experiments using primary melanoma explants cultured in three-dimensional collagen lattices have shown that decrease in cell-cell contacts leads to conversion of multicellular migration to single-cell movement. ${ }^{17}$ It has been shown that, in addition to loss of cell growth control, invasion appears to require an imbalance in the regulation of cell motility. ${ }^{18}$ It is plausible that the observed dyscohesion between melanoma cells corresponds to such a shift in the motility properties of the invasive tumour. Such transformation might favour dissociation of the malignant melanocytes from their cluster and acquisition of single-cell movement.

Interestingly, the majority of the melanomas examined in this study often showed intratumoural variation in cell cohesion among different subpopulations of malignant cells. This might be related to the well-known heterogeneity of malignant tumours with respect to several phenotypic characteristics, including metastatic ability. It has been shown that tumours initially containing predominantly poorly metastatic cells may eventually develop dominant clones of cells with metastatic potential. ${ }^{19}$ As reduction of homotypic interactions is one of the features of metastatic potential, it can be hypothe- sized that in melanomas with high dyscohesion scores, clones of malignant melanocytes with increased metastatic potential have become dominant. This could explain how the percentage of dyscohesive cells achieves prognostic significance.

It is possible that the observed variation in melanoma cell cohesion may reflect corresponding differences in the expression of adhesion molecules, especially cadherins, the principal agents for homotypic cell-cell adhesion. A model of shift in cadherin expression during melanoma tumour progression from radial growth phase to metastatic disease has been proposed by Herlyn and Gruss. ${ }^{19}$ According to this model, in radial growth phase, melanoma cells have downregulated E-cadherin expression and therefore acquired the potential to detach from the epidermal keratinocytes. In metastatic melanomas, they simultaneously start expressing $\mathrm{N}$-cadherin, which provides them with the ability for homotypic interactions with dermal mesenchymal cells and endothelial cells also expressing this cadherin subtype. These interactions have been postulated as critical for rendering melanoma cells capable of invasion and metastasis. ${ }^{19}$

In vivo studies have shown that in normal epidermis, the scattered melanocytes interact with adjacent keratinocytes by E-cadherinmediated homotypic interaction ${ }^{10}$ and normal melanocytes express E-cadherin in vitro. ${ }^{10,20}$ Conversely, 

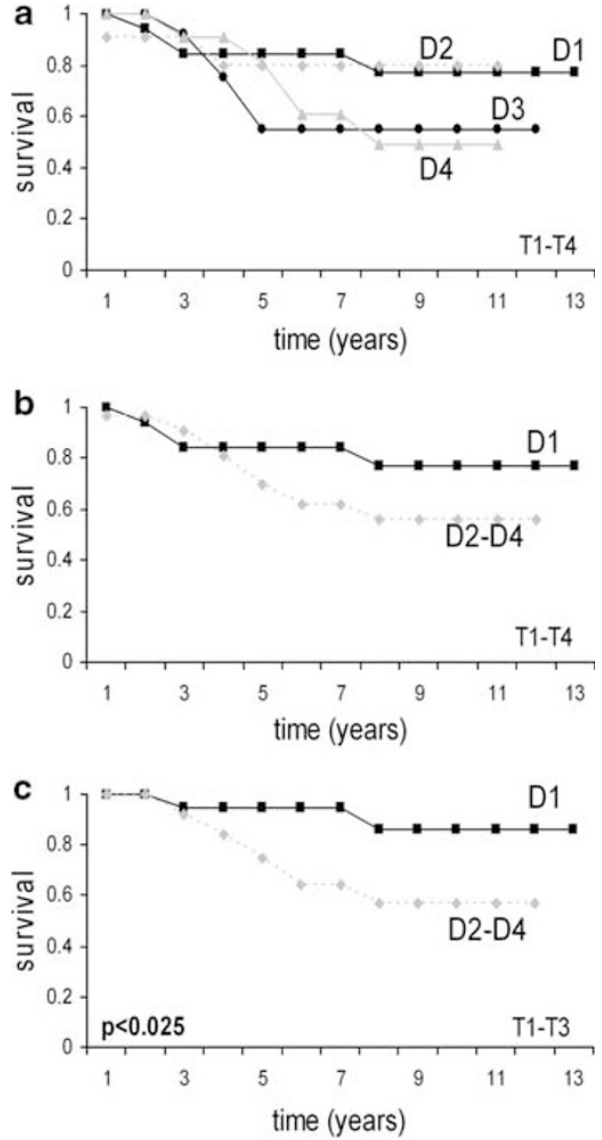

Figure 6 (a) Kaplan-Meier survival curves of all 75 melanomas comparing lesions with dyscohesion scores 1-4 (D1-D4). (b) Kaplan-Meier survival curves of all 75 melanomas comparing lesions with dyscohesion score 1 (D1) with lesions with dyscohesion scores 2-4 (D2-D4) pooled together. The difference was not significant. (c) Kaplan-Meier survival curves of T1-T3 melanomas only comparing lesions with dyscohesion score 1 (D1) with lesions with dyscohesion scores 2-4 (D2-D4) pooled together. The difference was significant $(P<0.025$, log-rank test).

heterogeneous and variable E-cadherin expression has been reported in melanomas not only between samples but within the same tumour as well. ${ }^{21-24} \mathrm{We}$ postulate that such a variability of expression might reflect the proportion of dyscohesive cells in their dermal component. We are currently pursuing this hypothesis by studying the spatial expression of E-cadherin (areas of dyscohesion compared to areas of preservation of cohesion) in melanomas with different dyscohesion scores. Interestingly, in breast carcinomas cells expressing E-cadherin were mixed with cells not expressing it, ${ }^{25-27}$ with the latter located mainly in areas composed of poorly cohesive cells. ${ }^{27}$

As it is difficult to estimate the degree of intercellular connection from the expression of a single adhesion molecule, purely morphological evaluation of cohesion might possess the advantage of assessing the outcome of the orchestrated contribution of several adhesion molecules. For example, $\beta 1$ integrins have been also shown to have a function in melanoma cell cohesion and patterns of migration in vitro. ${ }^{17}$ This might be an explanation for the fact that the correlation between morphologically assessed cohesion and prognosis in melanomas is stronger than previous attempts to correlate immunohistochemically detected adhesion molecule expression on melanoma cells with prognosis., ${ }^{21,23}$ It could, therefore, be argued that morphological evaluation of tumour cell cohesion may be a simple, cost-effective technique to predict the biologic behaviour of melanomas.

Our data show that melanoma thickness is not related to dyscohesion and $\mathrm{T} 1$ melanomas have similar distribution of dyscohesion scores as thicker ones. Interestingly, the hazard ratios of thinner melanomas were lower with none of the five T1 melanomas with dyscohesion score 1 showing metastasis. In contrast, three out of six cases of T1 melanomas with dyscohesion scores 2-4 presented with metastasis. It has been previously reported that $2-18 \%$ of very thin melanomas $(\leq 0.75 \mathrm{~mm})$ relapse over 0-11 years. ${ }^{15}$ However, reliable histological parameters predicting such behaviour remain elusive. ${ }^{6,15}$ We are currently pursuing the assessment of cell cohesion in a larger series of thin melanomas, including cases with Breslow thickness $\leq 0.75 \mathrm{~mm}$, with the aim of identifying subgroups of patients with potentially aggressive thin melanomas. Conversely, a possible explanation of the increasing hazard ratios going from $\mathrm{T} 1$ to $\mathrm{T} 4$ melanomas might be that in thicker melanomas even a minor proportion of dyscohesive dermal component translates, in absolute terms, as a substantial mass of dyscohesive tumour. Alternatively, as already suggested for the prognostic significance of breast carcinoma cell dyscohesion, ${ }^{28}$ melanomas above a particular thickness threshold may have a high rate of local metastasis regardless of the degree of tumour cell cohesion.

Interestingly, cell cohesion was not only unrelated to Breslow thickness but in our cohort of patients, appears to be a more potent prognostic parameter than the latter. This highlights the fact that acquisition of metastatic potential is not necessarily related to tumour size or proliferation rate.

In our cohort, Breslow thickness was not significantly correlated with disease-free survival, clearly possibly due to small number of cases. However, there was a progressive decline in the Kaplan-Meier curves from $\mathrm{T} 1$ to $\mathrm{T} 4$ tumours. Ulceration, a known independent prognostic factor, ${ }^{8}$ was significantly correlated with a lower disease-free survival. In contrast, regression, the presence of which has been shown to increase the risk of metastasis, ${ }^{3}$ did not show any significant correlation with disease-free survival.

In conclusion, we have found that the degree of melanoma cell dyscohesion is a reliable histological prognostic factor unrelated to tumour thickness, regression or ulceration. This may be applicable in clinical practice as there appears to be low 
inter-observer variation. However, before recommendation for assessment of cellular cohesion in clinical practice, studies with larger samples are required.

It has been emphasized that tumour cell invasion and metastasis is the orchestrated end-result of interactions between gene products that promote it versus others suppressing it. Such processes are of a dynamic nature and take place in discrete microenvironments within a tumour. ${ }^{9}$ We believe that techniques facilitating the in situ study of molecular events within distinct microenvironments in tissue can be used for the clarification of such cellular processes.

\section{Disclosure/conflict of interest}

The authors declare no conflict of interest.

\section{References}

1 Dvir E, Gellei B, Hirshowitz B. Measurement of tumor thickness as a prognostic tool in primary malignant melanoma of the skin. Ann Plast Surg 1980;5:216-221.

2 Lang PG. Current concepts in the management of patients with melanoma. Am J Clin Dermatol 2002; 3:401-426.

3 Slominski A, Wortsman J, Carlson AJ, et al. Malignant melanoma. An update. Arch Pathol Lab Med 2001;125: 1295-1306.

4 Clark Jr WH, Elder DE, Guerry D, et al. A study of tumor progression: the precursor lesions of superficial spreading and nodular melanoma. Hum Pathol 1984;15:1147-1165.

5 Wick MP. Prognostic factors for cutaneous melanoma. Am J Clin Pathol 1998;110:713-718.

6 Cochran AJ, Bailly C, Luo F, et al. Prediction of outcome for patients with cutaneous melanoma. Curr Diagn Pathol 2003;9:302-312.

7 Breslow A. Problems in the measurement of tumor thickness and level of invasion in cutaneous melanoma. Hum Pathol 1977;8:1-2.

8 Balch CM, Buzaid AC, Soong S-J, et al. Final version of the American Joint Committee on Cancer Staging System for cutaneous melanoma. J Clin Oncol 2001;19: 3635-3648.

9 van Roy F, Mareel M. Tumour invasion: effects of cell adhesion and motility. Trends Cell Biol 1992;2: 163-169.

10 Hsu M-Y, Wheelock MJ, Johnson KR, et al. Shifts in cadherin profiles between human normal melanocytes and melanomas. J Investig Dermatol Symp Proc 1996;1: 188-194.

11 Layfield LJ. Fine needle aspirate smear morphology in metastatic melanoma. Acta Cytol 1988;33:606-612.
12 Reed RJ. The histological variance of malignant melanoma: the interrelationship of histological subtype, neoplastic progression, and biological behaviour. Pathology 1985;17:301-312.

13 Nakhleh RE, Wick MR, Rocamora A, et al. Morphologic diversity in malignant melanomas. Am J Clin Pathol 1990;93:731-740.

14 Smolle J, Hofmann-Wellenhof $\mathrm{R}$, Kofler $\mathrm{R}$, et al. Computer simulations of histologic patterns in melanoma using a cellular automaton provide correlations with prognosis. J Invest Dermatol 1995;105:797-801.

15 Fearfield LA, Rowe A, Francis N, et al. Clinicopathological features of relapsing very thin melanoma. Clin Exp Dermatol 2001;26:686-695.

16 Gabbert H. Mechanisms of tumor invasion: evidence from in vivo observations. Cancer Metastasis Rev 1985;4:293-309.

17 Hegerfeldt Y, Tusch M, Bröcker E-B, et al. Collective cell movement in primary melanoma explants: plasticity of cell-cell interaction, $\beta 1$-integrin function, and migration strategies. Cancer Res 2002;62:2125-2130.

18 Liotta LA, Steeg PS, Stetler-Stevenson WG. Cancer metastasis and angiogenesis: an imbalance of positive and negative regulation. Cell 1991;64:327-336.

19 Gruss C, Herlyn M. Role of cadherins and matrixins in melanoma. Curr Opin Oncol 2001;13:117-123.

20 Seline PC, Norris DA, Horikawa T, et al. Expression of $\mathrm{E}$ and P-cadherin by melanoma cells decreases in progressive melanomas and following ultraviolet radiation. J Invest Dermatol 1996;106:1320-1324.

21 Silye R, Karayiannakis AJ, Syrigos KN, et al. E-cadherin/catenin complex in benign and malignant melanocytic lesions. J Pathol 1998;186:350-355.

22 Sanders DSA, Blessing K, Hassan GAR, et al. Alterations in cadherin and catenin expression during the biological progression of melanocytic tumours. Mol Pathol 1999;52:151-157.

23 Danen EHJ, de Vries TJ, Morandini R, et al. E-cadherin expression in human melanoma. Melanoma Res 1996;6:127-131.

24 Cowley GP, Smith MEF. Cadherin expression in melanocytic naevi and malignant melanomas. J Pathol 1996;179:183-187.

25 Sauer T, Boudjema G, Jebsen PW, et al. Immunocytochemical expression of E-cadherin on fine-needle aspirates from breast carcinomas correlate with the cell dissociation pattern seen on smears. Diagn Cytopathol 2001;25:382-388.

26 Oka H, Shiozaki H, Kobayashi K, et al. Expression of E-cadherin cell adhesion molecules in human breast cancer tissues and its relationship to metastasis. Cancer Res 1993;53:1696-1701.

27 Gamallo C, Palacios J, Suarez A, et al. Correlation of Ecadherin expression with differentiation grade and histological type in breast carcinoma. Am J Pathol 1993;142:987-993.

$28 \mathrm{Yu} \mathrm{GH}$, Cajulis RS, DeFrias DVS. Tumor cell (dys)cohesion as a prognostic factor in aspirate smears of breast carcinomas. Am J Clin Pathol 1998;109: 315-319. 